\title{
Effectiveness of community-based rehabilitation interventions incorporating outdoor mobility on ambulatory ability and falls-related self-efficacy after hip fracture: a systematic review and meta-analysis
}

\author{
Katie J. Sheehan ${ }^{1} \cdot$ Laura Fitzgerald $^{1} \cdot$ Kate Lambe $^{1} \cdot$ Finbarr C. Martin $^{1} \cdot$ Sallie E. Lamb ${ }^{2} \cdot$ Catherine Sackley $^{1}$
}

Received: 9 March 2021 / Accepted: 23 May 2021 / Published online: 19 June 2021

(c) The Author(s) 2021

\begin{abstract}
Summary There is limited evidence from 11 randomised controlled trials on the effect of rehabilitation interventions which incorporate outdoor mobility on ambulatory ability and/or self-efficacy after hip fracture. Outdoor mobility should be central (not peripheral) to future intervention studies targeting improvements in ambulatory ability.

Purpose Determine the extent to which outdoor mobility is incorporated into rehabilitation interventions after hip fracture. Synthesise the evidence for the effectiveness of these interventions on ambulatory ability and falls-related self-efficacy.

Methods Systematic search of MEDLINE, Embase, PsychInfo, CINAHL, PEDro and OpenGrey for published and unpublished randomised controlled trials (RCTs) of community-based rehabilitation interventions incorporating outdoor mobility after hip fracture from database inception to January 2021. Exclusion of protocols, pilot/feasibility studies, secondary analyses of RCTs, nonrandomised and non-English language studies. Duplicate screening for eligibility, risk of bias, and data extraction sample. Random effects meta-analysis. Statistical heterogeneity with inconsistency-value $\left(\mathrm{I}^{2}\right)$.

Results RCTs $(n=11)$ provided limited detail on target or achieved outdoor mobility intervention components. There was conflicting evidence from 2 RCTs for the effect on outdoor walking ability at 1-3 months (risk difference $0.19 ; 95 \%$ confidence intervals (CI): $0.21,0.58 ; \mathrm{I}^{2}=92 \%$ ), no effect on walking endurance at intervention end (standardised mean difference 0.05 ; $95 \% \mathrm{CI}:-0.26,0.35 ; \mathrm{I}^{2}=36 \%$ ); and suggestive (CI crosses null) of a small effect on self-efficacy at $1-3$ months (standardised mean difference $0.25 ; 95 \% \mathrm{CI}:-0.29,0.78 ; \mathrm{I}^{2}=87 \%$ ) compared with routine care/sham intervention.

Conclusion It was not possible to attribute any benefit observed to an outdoor mobility intervention component due to poor reporting of target or achieved outdoor mobility and/or quality of the underlying evidence. Given the low proportion of patients recovering outdoor mobility after hip fracture, future research on interventions with outdoor mobility as a central component is warranted.
\end{abstract}

Trial registration PROSPERO registration: CRD42021236541

Keywords Physiotherapy $\cdot$ Walking $\cdot$ Falls efficacy $\cdot$ Fracture neck of femur $\cdot$ Home-based

Katie J. Sheehan

katie.sheehan@kcl.ac.uk

1 Department of Population Health Sciences, School of Population and Environmental Sciences, Kings College London, London, UK

2 Institute of Health Research, University of Exeter, Devon, UK

\section{Introduction}

Each year, United Kingdom (UK) hospitals admit 70,000 older adults with hip fracture [1]. Even with surgery, there is a fivefold to eightfold increased risk for all-cause mortality in the first 3 months after hip fracture [2]. Among survivors, only $34 \%$ regain their pre-fracture mobility (ability to move from and between different postures, e.g. sitting, standing, and walking) by 6-month post-fracture [3]. This may contribute to the reported high rates of transition from independent living to nursing homes among persons with hip fracture $[4,5]$. The observed increases in mortality and morbidity led 81 global societies to endorse a call to action for 
ongoing post-acute care of people whose ability to function is impaired by hip and other major fragility fractures [6].

In a UK qualitative study, patients who were mobile prior to hip fracture identified stability, avoiding falls, and not being afraid of falls during meaningful activities as the outcomes they valued most during their recovery [7]. Indeed, high falls-related self-efficacy and the physical ability to mobilise outdoors are critical outcomes to enable participation in social and family networks and activities [8]. However, up to $65 \%$ of older adults report low falls-related selfefficacy after hip fracture [9], and a recent analysis of 24,492 patients indicated a weighted probability of up to $10 \%$ for recovery of mobility at 30 days among those able to walk outdoors pre-fracture [10].

To achieve benefits in terms of falls-related self-efficacy and outdoor mobility, a rehabilitation intervention should be tailored to explicitly target improvements in these outcomes [11]. Indeed, a 2010 review reported a potential benefit of psychological intervention on self-efficacy after hip fracture from two RCTs [12]. A previous systematic review identified nine randomised controlled trials (RCTs) of homebased rehabilitation interventions after hip fracture [13]. The authors concluded home-based rehabilitation had considerable positive effect on physical functioning after hip fracture but no effect on walking outdoors [13]. The authors did not describe the extent to which outdoor mobility was incorporated into the home-based rehabilitation interventions identified by their review [13]. Outdoor mobility is likely more physically (gait, strength, and balance), psychologically (confidence, falls-related self-efficacy), and cognitively (navigating environments) challenging than indoor mobility [14]. It is therefore not clear whether the lack of effectiveness was due to an absence of outdoor mobility intervention components across RCTs included in the review [13]. This uncertainty translated to an absence of guidance for interventions to improve falls-related self-efficacy and outdoor mobility after hip fracture in national guidelines $[15,16]$.

We sought to address this evidence gap by:

1. Determining the extent to which outdoor mobility is incorporated into rehabilitation interventions after hip fracture; and

2. Synthesising the evidence for the effectiveness of these interventions on ambulatory ability (outdoor walking and endurance) and falls-related self-efficacy.

\section{Methods}

\section{Protocol and registration}

This review is reported in adherence to the Preferred Reporting Items for Systematic Review and Meta-analysis statement [17]. The protocol is registered on the International Register of Systematic Reviews (PROSPERO CRD42021236541).

\section{Eligibility criteria}

We included randomised controlled trials (RCT) of community-based rehabilitation interventions which incorporated outdoor mobility for persons after hip fracture. Rehabilitation was defined as 'a set of interventions designed to optimize functioning and reduce disability in individuals with health conditions in interaction with their environment' [18]. Rehabilitation interventions for participants after hip fracture are often complex incorporating several interacting components. We employed a broad definition of 'outdoor mobility' to determine the extent to which outdoor mobility was captured by these components. This definition included components which targeted going outdoors for structured/ unstructured exercise/activity to those which targeted going outdoors for participation, e.g. taking public transport. We included RCTs which planned to incorporate supervised outdoor mobility, unsupervised outdoor mobility, and/or encouragement of outdoor mobility irrespective of whether this was completed by all participants within the RCT. We included RCTs irrespective of comparator group, outcomes measured, length of follow-up, and publication year. We excluded protocols, pilot/feasibility studies, secondary analyses of RCTs, and nonrandomised studies. We excluded RCTs not published in English, due to lack of resources for expert translation.

\section{Information sources}

We searched MEDLINE, Embase and PsychInfo (OVID), CINAHL (EBSCOhost), PEDro, and OpenGrey for published and unpublished RCTs from database inception to 13 January 2021.

\section{Search}

We used a published search strategy based on population, intervention, and study design (hip fracture, rehabilitation, and randomised controlled trials) limited to human and English language (Supplementary File 1) [19].

\section{Study selection}

Three reviewers screened title and abstracts (R1, R2, R3), and two reviewers screened full texts (R1, R3) of potentially eligible RCTs against eligibility criteria. Conflicts were resolved by consensus. Cohen's Kappa was estimated at $\mathrm{k}=0.7$ (moderate agreement) for inter-rater reliability prior to consensus work of full-text screening [20]. 


\section{Data collection process and data items}

Two reviewers $(\mathrm{R} 1, \mathrm{R} 2)$ piloted data extraction onto a template adapted from the taxonomy to classify and describe fall-prevention interventions [21]. We sought data for the following data items: author, year, location, sample size intervention group, sample size control group; approach - aim, inclusion criteria, exclusion by dementia/cognitive impairment, other exclusion; base - recruitment, site (s) of delivery, assessment delivered by, intervention delivered by; components - assessment as part of intervention, combination of interventions and description; descriptor intervention - supervised/unsupervised (type, duration, frequency, intensity, individual/group), psychological (cognitive behavioural therapy, other, individual/group), environment, assistive technology, knowledge, postintervention follow-up (period, type, completeness) and strategies to improve uptake/adherence; descriptor control - routine care/no specific intervention, supervised exercises, medication, knowledge, social environment and other; and outcome - primary, secondary, effect for primary outcome at intervention end and intervention followup. Following the pilot, an additional data item specifically related to outdoor mobility was added. One reviewer (R2) extracted the remaining data onto the template. Final extraction was checked for accuracy by a second reviewer (R1). We extracted data from the earliest publication where multiple publications referred to one RCT.

\section{Risk of bias in individual studies}

Two reviewers independently assessed risk of bias at the study level using the Cochrane Risk of Bias Tool (R1, R2) [22]. Conflicts were resolved by consensus.

\section{Synthesis of results}

For our first objective, we reported the extent to which outdoor mobility was incorporated into rehabilitation interventions in a narrative synthesis. For our second objective, we completed an inverse variance random effects meta-analysis to estimate standardised mean difference (for continuous outcomes) or risk difference (for binary outcomes) and their $95 \%$ confidence intervals. We interpreted a standardised mean/risk difference of $<0.2$ as null, $0.2-0.49$ as small, $0.5-0.79$ as medium and $\geq 0.8$ as large [23]. Statistical heterogeneity was evaluated using the inconsistency-value $\left(\mathrm{I}^{2}\right)$. Results of meta-analysis were presented in tables and forest plots. Meta-analyses were completed in RevMan Version 5.3 (Copenhagen: The Nordic Cochrane Centre, The Cochrane Collaboration, 2011).

\section{Risk of bias across studies}

Small-study publication bias was evaluated through interpretation of funnel plots for each outcome.

\section{Results}

\section{Selection}

We identified 5681 articles after de-duplication. We excluded 5569 on abstract screening. We excluded 99 on full-text screening for nonrandomised study design $(n=31)$, population $(n=10)$, intervention $(n=55)$, language $(n=2)$, no response from author for additional data related to eligibility $(\mathrm{n}=2)$ and leaving 12 papers reporting 11 RCTs (Fig. 1).

\section{Risk of bias within studies}

Most RCTs were at low risk of bias for random sequence generation $(n=10)$, blinding of outcome assessor $(n=8)$, incomplete outcome data $(n=8)$ or selective reporting $(n=11)$ (Fig. 2). There was insufficient information to assess allocation concealment for 7 RCTs. Lack of blinding of personnel and participants was the most common reason for high bias assignment $(n=5)$ [24-29]. In addition, one RCT did not blind outcome assessors [24, 25].

\section{Characteristics of included RCTs}

Detailed characteristics for the 11 RCTs are available in Table 1. RCTs were completed in Australia $(n=1)$ [29], Finland $(n=1)$ [30], Germany $(n=2)[26,31]$, the Netherlands $(n=1)$ [32], Sweden $(n=2)[24,25,28]$ and the USA $(n=4)$ [27, 33-35]. Sample size ranged from 28 [31] to 240 participants [32]. Participants were older adults (eligible age range from 60 years plus [26, 30, 33] to 75 years plus [31]) admitted with hip fracture and treated surgically. Nine RCTs excluded potential participants based on their cognitive function [24-27, 29-32, 34, 35]. Karlsson et al. [28] explicitly stated inclusion of participant irrespective of cognitive status, whilst Magaziner et al. excluded participants with 'low potential to benefit' or 'practical impediments to participation' [33]. Participants were recruited from acute hospital [24, 25, 28, 29, 34, 35], inpatient rehabilitation [26, 31], clinic/health centres [27,33], nursing and community care facilities [32] or the community [30]. Outcome assessments were completed by physiotherapists [27, 30, 33], occupational therapist [24, 25, 32], gerontologist and psychologist [26], researchers [28] or were not specified [29, 31, 34].

Seven RCTs compared interventions to routine care. This routine care was described as inpatient services, pathways 
Fig. 1 Study selection
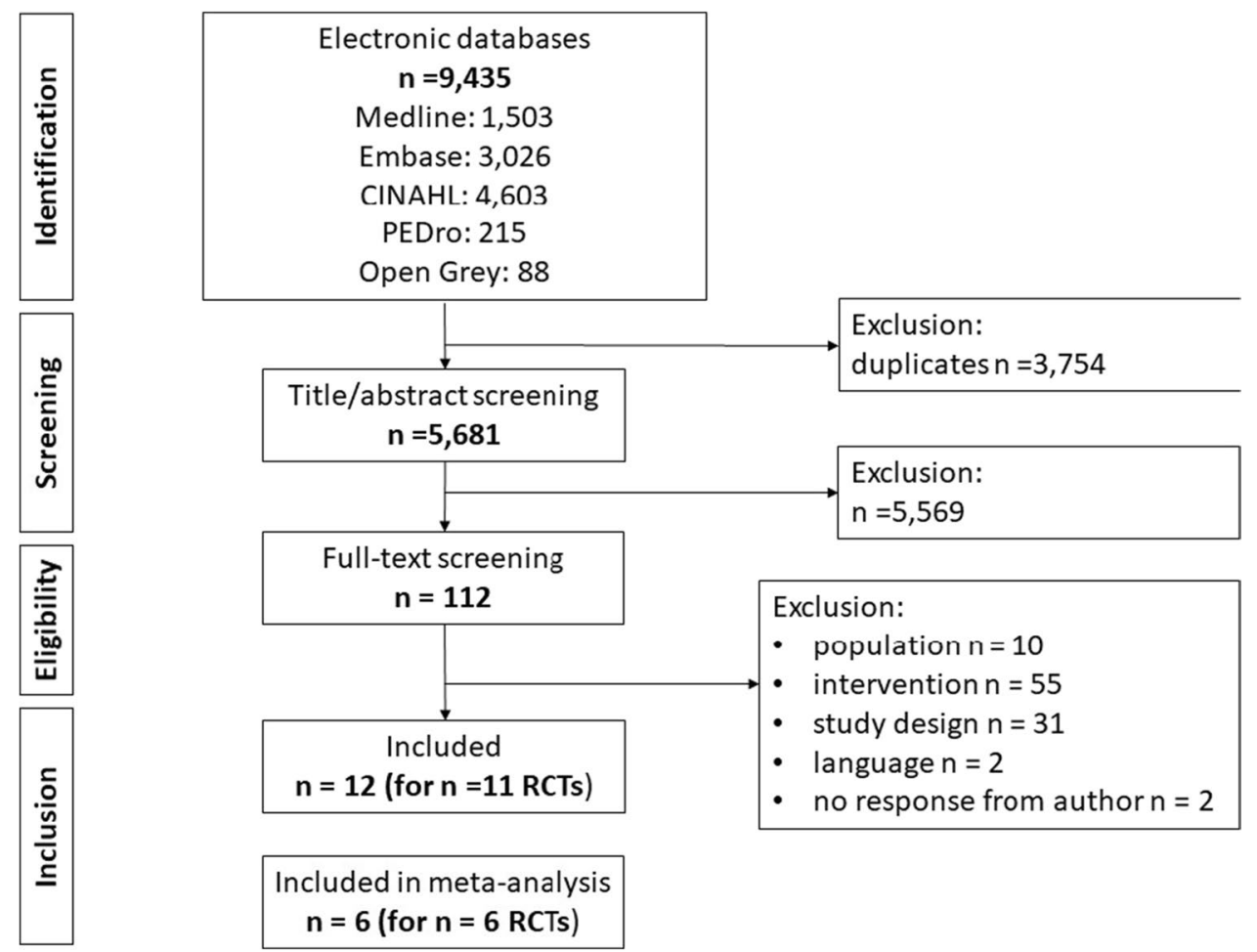

Fig. 2 Risk of bias

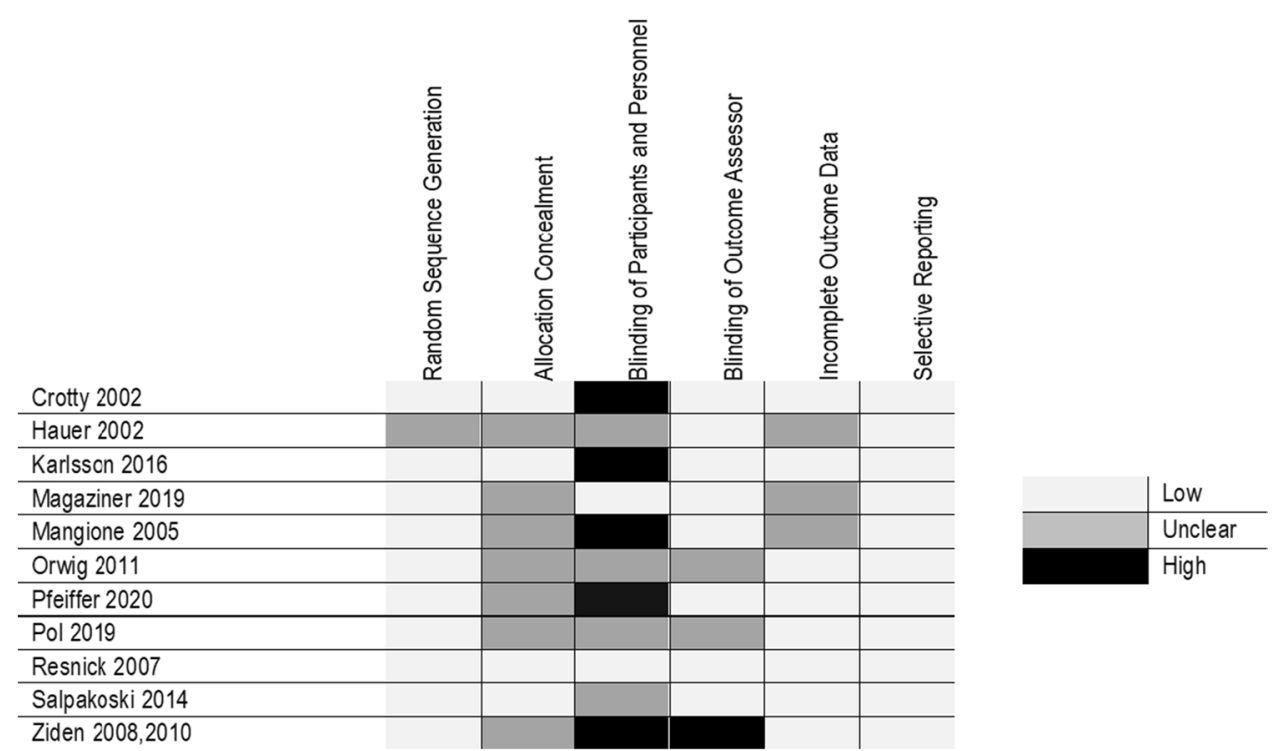

and discharge planning [29]; inpatient rehabilitation for 2-4 weeks [26, 34]; inpatient rehabilitation based on functional needs and a single home therapy evaluation [35]; or interdisciplinary inpatient rehabilitation, discharge planning, referral to ongoing outpatient rehabilitation [24, 25, $28,32]$ including handover to physiotherapists/occupational therapists at residential care facilities [28]. Two RCTs provided written materials (home exercise programme [30], non-exercise related written materials [27]) with no further follow-up. Two interventions were compared to sham active controls including seated activities [31], or seated activities and transcutaneous electrical stimulation [33]. Detailed descriptions for each intervention are available in Table 2.

\section{Synthesis: outdoor mobility in interventions}

All 11 RCTs included in this review included outdoor mobility in their intervention. This was explicitly stated by 6 RCTs [24-28, 32, 33] and confirmed with authors for the remaining 5 RCTs [29-31, 34, 35]. Outdoor mobility 


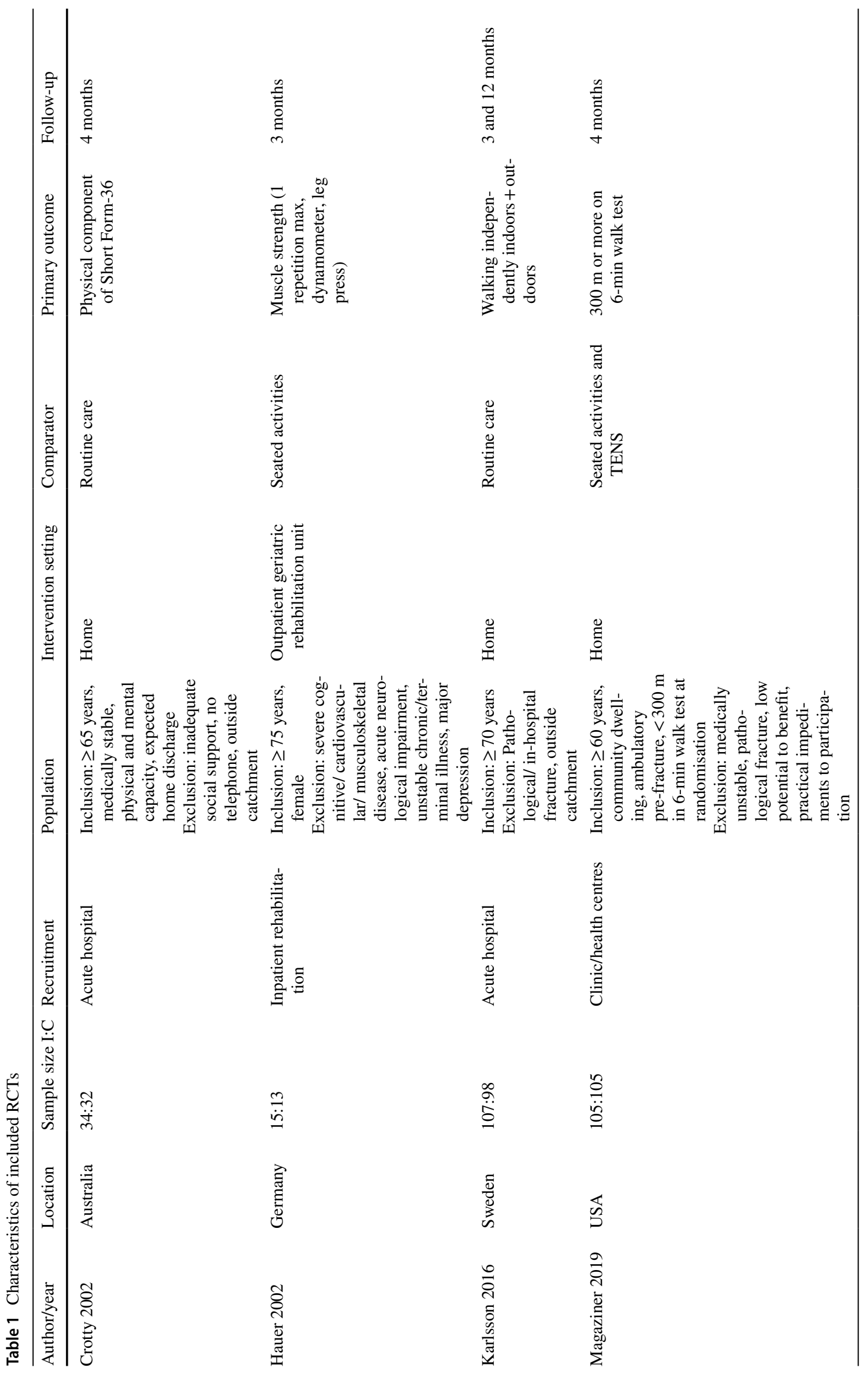




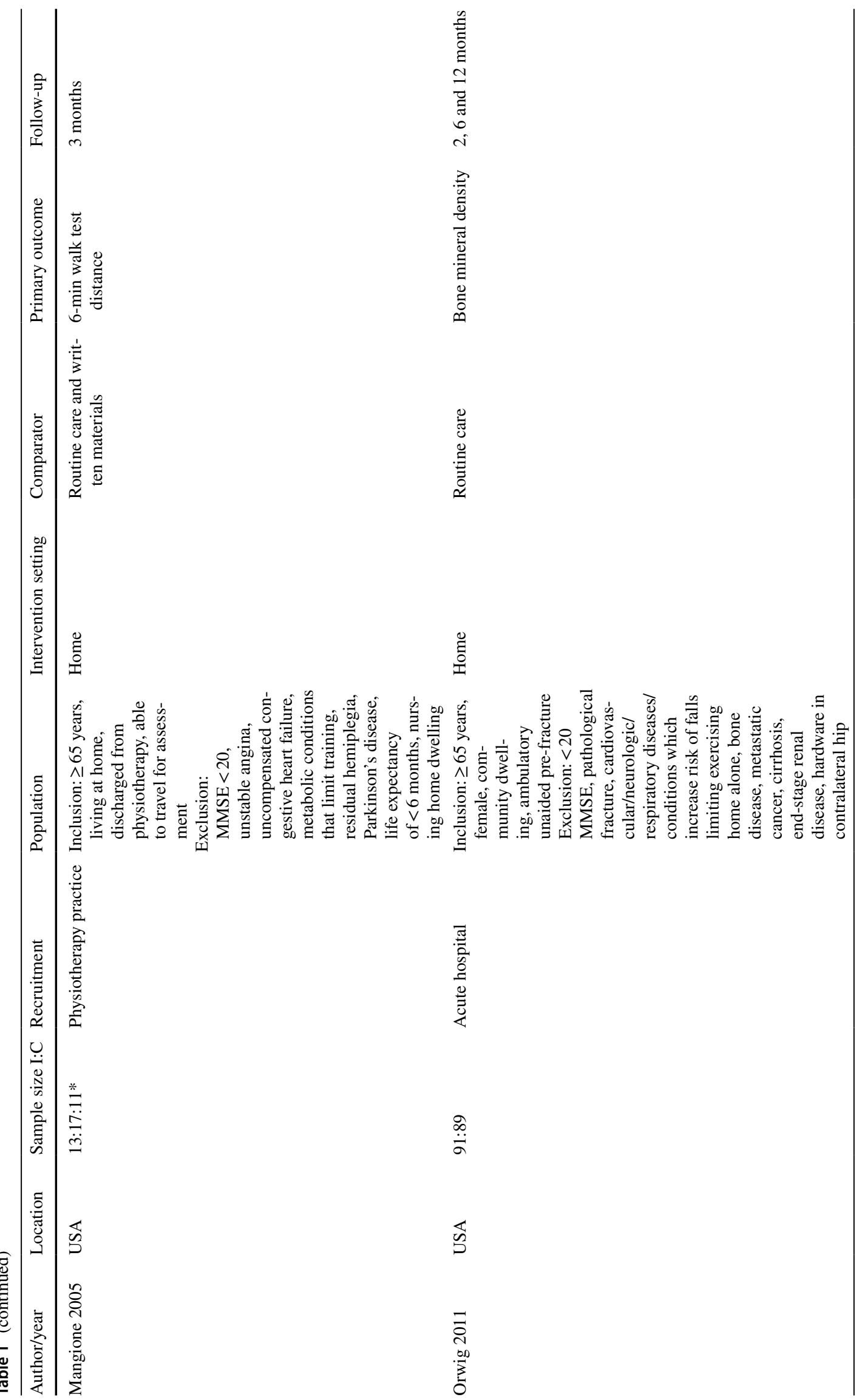




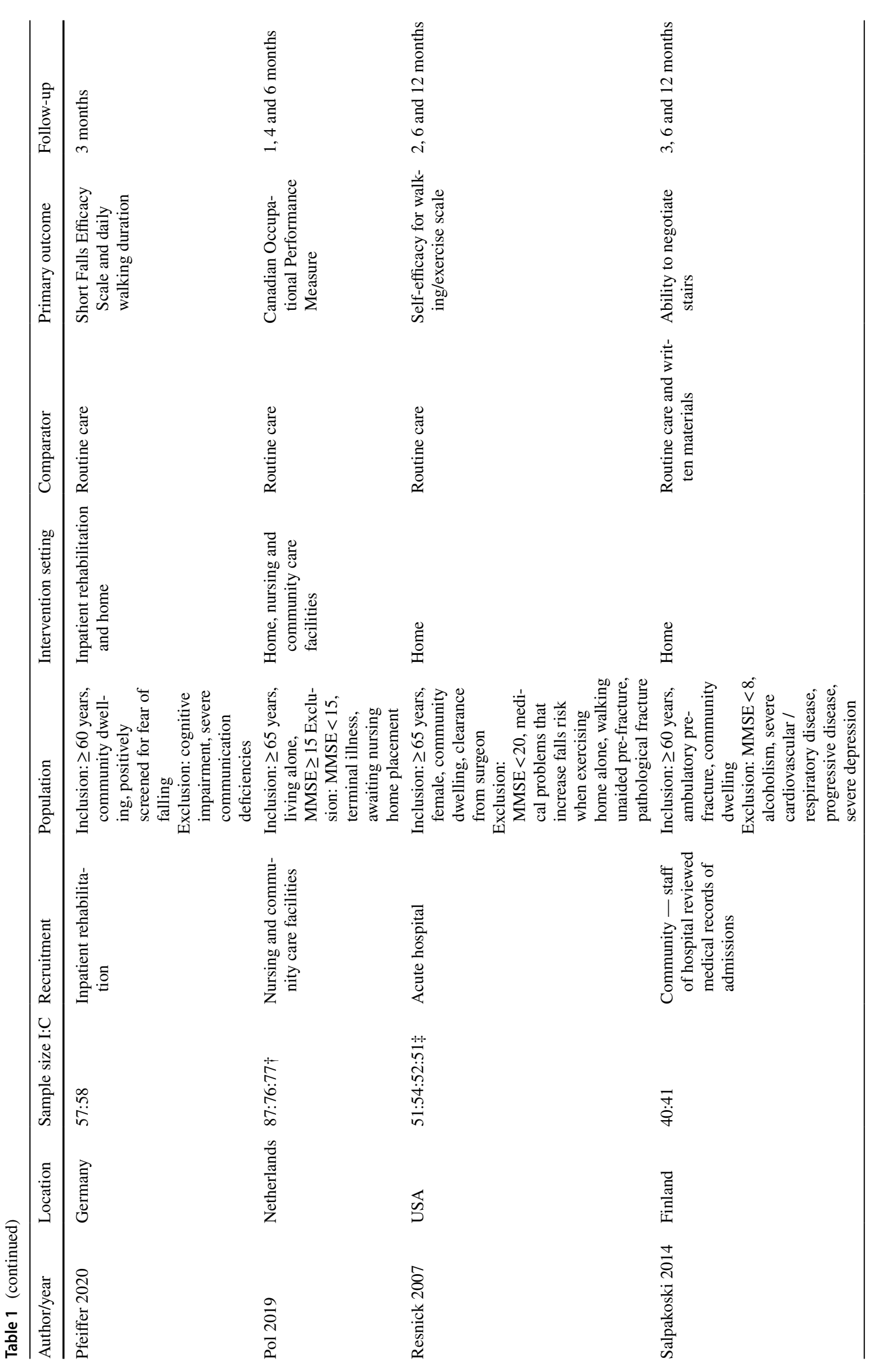




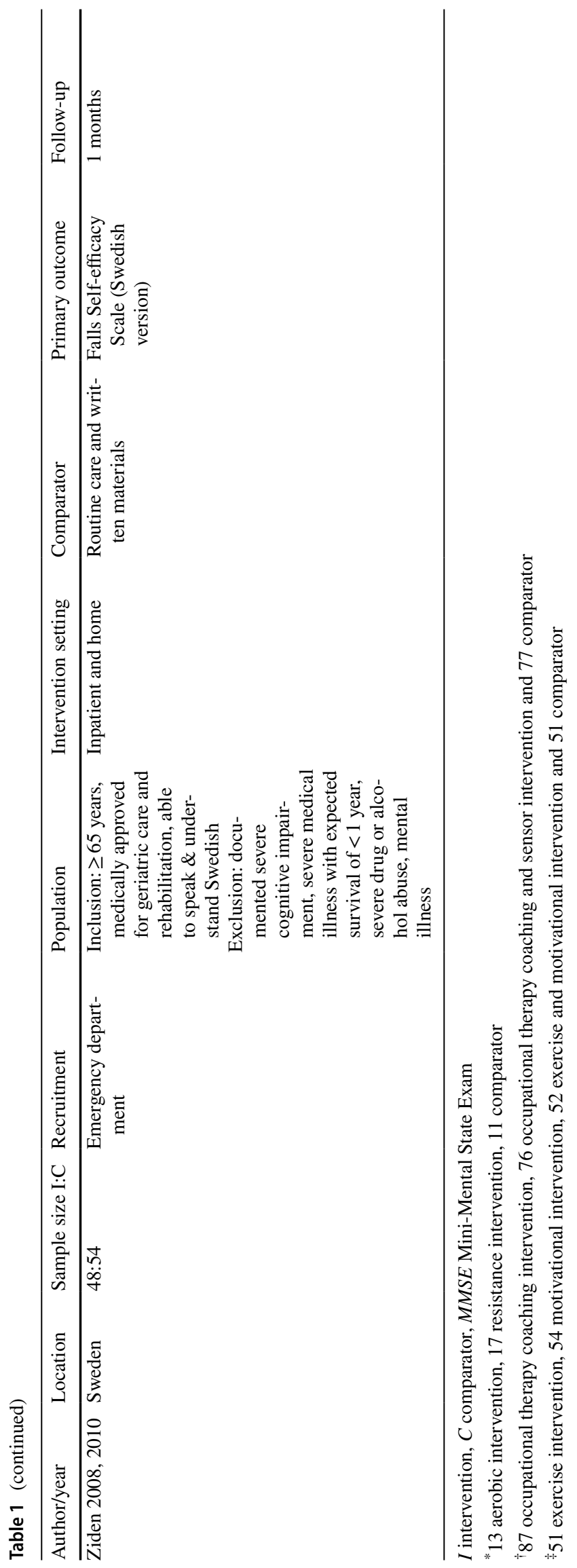

照 Springer 


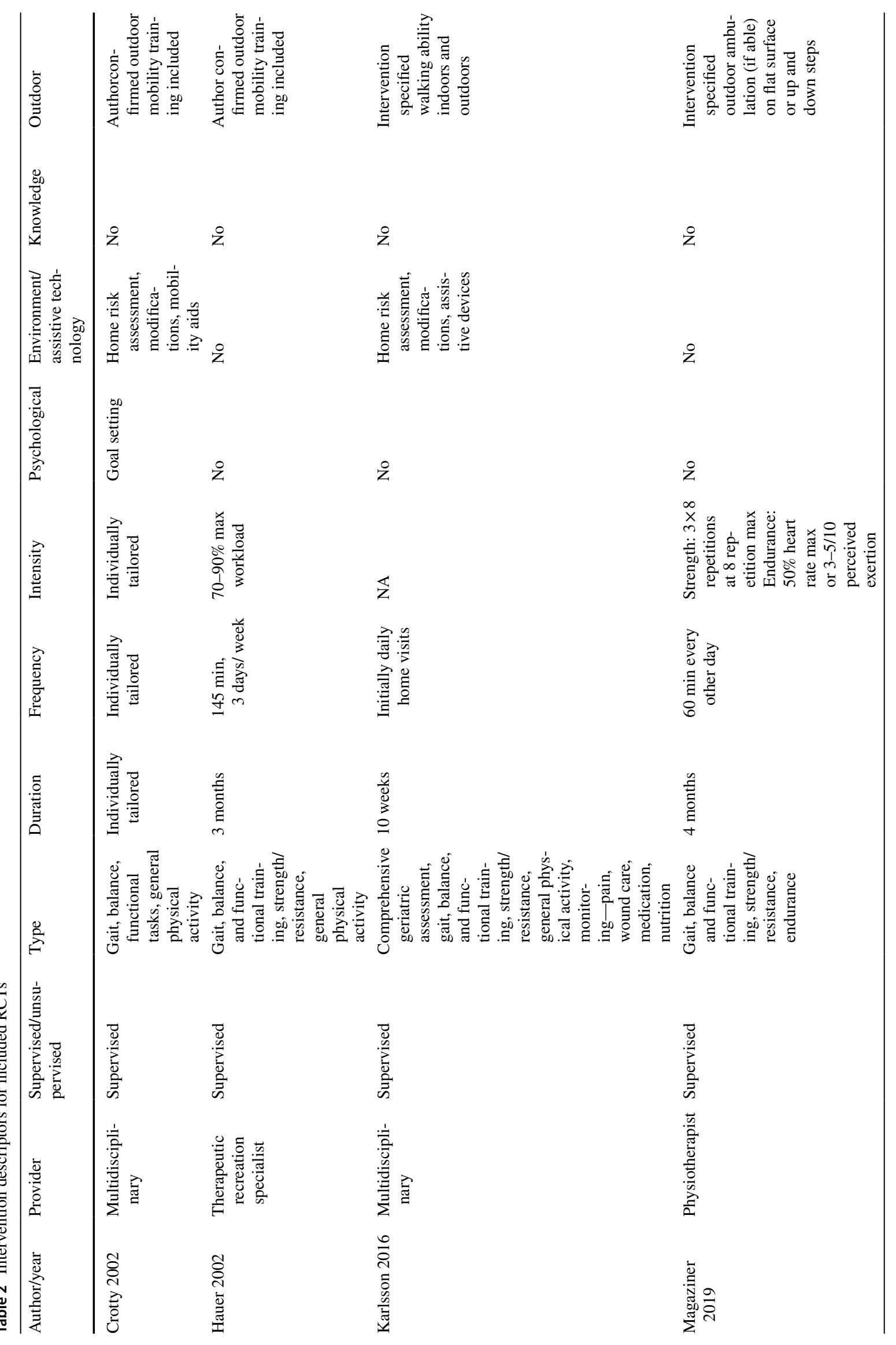




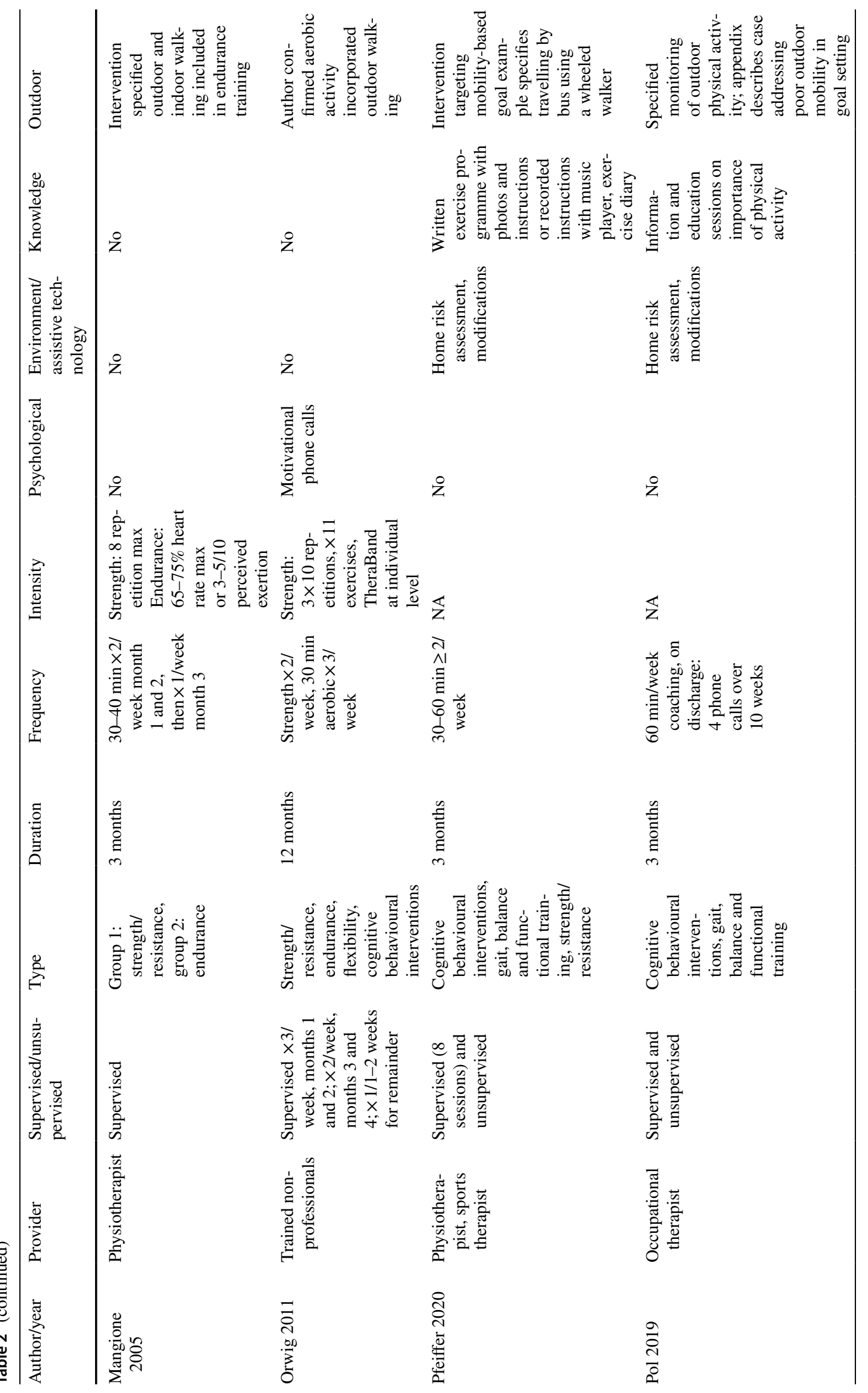




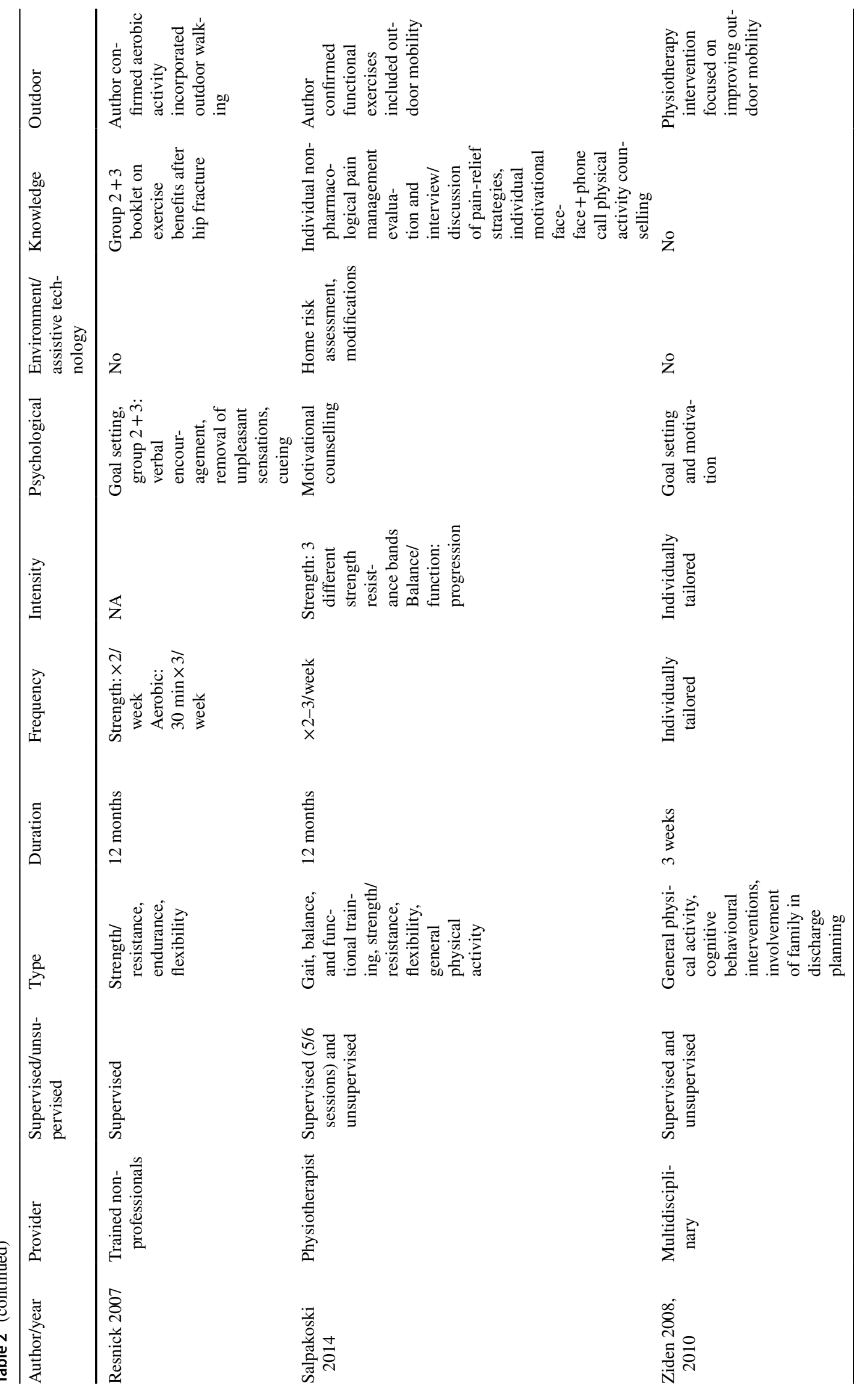


was supervised [27-29, 31, 33, 35], unsupervised [26, 32] or both supervised and unsupervised [24, 25, 30, 34]. The target duration/distance, frequency, type (e.g. using transport) and/or intensity of outdoor mobility (independent of indoor mobility) was not described for any RCT included in this review. Two authors provided data on the extent to which outdoor mobility was achieved by participants in their RCT $[27,33]$. Mangione indicated $83 \%$ of participants performed outdoor mobility during the intervention [27]. Crosssectional data presented at the American Physical Therapy Association, Combined Sections Meeting in 2019 by Mangione et al. [36], indicated the proportion of participants in the larger trial by Magaziner et al. [33] of home exercise after hip fracture who performed outdoor mobility during the home-delivered physical therapy intervention was as follows: visit 3, 44\% outdoor walking; visit $8,57 \%$ outdoor walking; visit $16,62 \%$ outdoor walking; visit $24,63 \%$ outdoor walking; and visit $32,56 \%$ outdoor walking (these are for different sample sizes and different seasons). The remaining RCTs did not detail the extent to which outdoor mobility as an intervention component was achieved by participants.

\section{Synthesis: intervention effectiveness}

There was no evidence of publication bias for any of the meta-analyses.

\section{Ambulatory ability}

\section{Outdoor walking}

Two RCTs selected outdoor walking as their primary outcome. Karlsson et al. defined outdoor walking as the ability to walk independently outdoors [28]. Ziden et al. defined outdoor walking as the ability to walk outdoor alone or with company [24, 25]. These RCTs reported conflicting evidence for the effect of rehabilitation interventions which incorporate outdoor mobility on outdoor walking ability at 1-3-month follow-up (risk difference $0.19 ; 95 \%$ confidence interval (CI): 0.21, 0.58) (Fig. 3) [24, 28]. There was substantial heterogeneity in the analysis $\mathrm{I}^{2}=92 \%$. This may be due to systematic differences in participants, interventions and/or target outcomes between the two trials. Karlsson et al. included participants with cognitive impairment and from residential care in their supervised intervention [28]. Ziden et al. excluded potential participants with cognitive impairment and from residential care from their supervised and unsupervised intervention which also incorporated psychological treatment components [24, 25]. Moreover, Ziden et al. explicitly targeted outdoor mobility in their intervention $[24,25]$.

At 12-month follow-up, there was no between-group difference in the proportion of patients who walked outdoors $[25,28]$. Karlsson et al. reported that $48.8 \%$ of participants in the intervention group walked outdoors compared to $48.7 \%$ of the comparator group, and $90 \%$ and $89 \%$ participants required a walking device for outdoor ambulation for intervention and comparator group respectively (increase from $69.2 \%$ and $65.3 \%$ at baseline) [28]. Ziden et al. reported the intervention group recovered outdoor walking by 1 -month follow-up, whereas the comparator group recovered outdoor walking by 6-month follow-up [25].

\section{Walking endurance}

Three RCTs selected walking endurance (6-min walk test [27, 33], walking time [26]) as their primary outcome. Rehabilitation interventions which incorporated outdoor mobility were not effective in improving walking endurance at intervention end (standardised mean difference $0.05 ; 95 \%$ CI: $-0.26,0.35$ ) (Fig. 4). There was low heterogeneity in the analysis $\mathrm{I}^{2}=36 \%$.

\section{Falls-related self-efficacy}

Three RCTs selected falls-related self-efficacy (Falls SelfEfficacy Scale (Swedish version) [24, 25], Short Falls Self-Efficacy Scale [26], self-efficacy for walking [35]) as their primary study outcome. One RCT included fallsrelated self-efficacy as a secondary study outcome noting a between-group difference at 4-month follow-up favouring the intervention group (median (25th and 75 th percentiles):

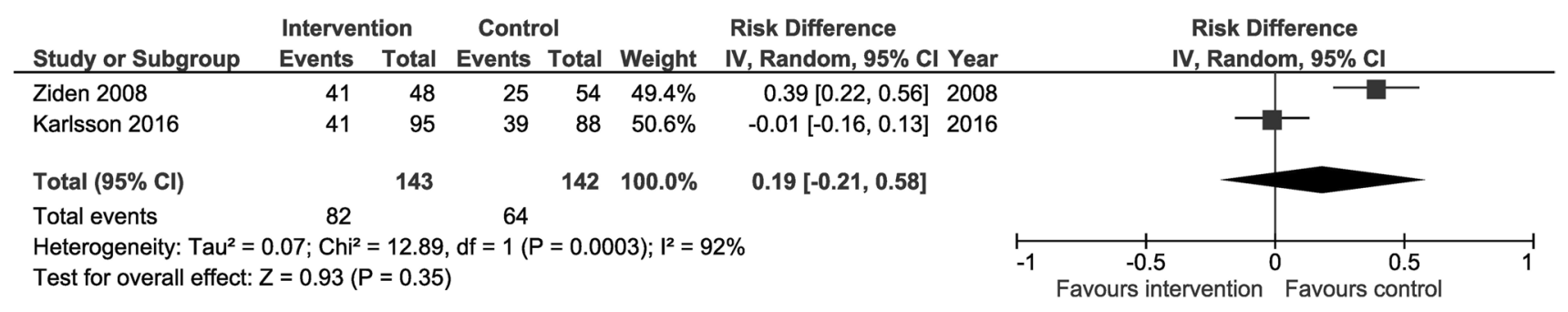

Fig. 3 Forest plot illustrating the standardised mean difference and 95\% confidence interval of outdoor walking at first follow-up (1-3 months) for rehabilitation interventions with outdoor mobility compared to routine care 


\begin{tabular}{|c|c|c|c|c|c|c|c|c|c|c|}
\hline \multirow[b]{2}{*}{ Study or Subgroup } & \multicolumn{3}{|c|}{ Intervention } & \multicolumn{3}{|c|}{ Control } & \multicolumn{2}{|c|}{ Std. Mean Difference } & \multirow{2}{*}{$\begin{array}{r}\text { Std. Mean Difference } \\
\text { IV, Random, } 95 \% \mathrm{CI}\end{array}$} & \\
\hline & Mean & SD & Total & Mean & SD & Total & Weight & IV, Random, 95\% Cl Year & & \\
\hline Mangione (Aerobic) 2005 & 321.1 & 101.7 & 12 & 266.2 & 82.4 & 10 & $11.1 \%$ & $0.56[-0.29,1.42] 2005$ & & \\
\hline Magaziner 2019 & 242.4 & 83.7 & 91 & 233.1 & 83.1 & 96 & $50.3 \%$ & $0.11[-0.18,0.40] 2019$ & - & \\
\hline Pfeiffer 2020 & 32.1 & 19.75 & 54 & 36.7 & 27.19 & 57 & $38.6 \%$ & $-0.19[-0.56,0.18] 2020$ & - & \\
\hline Total $(95 \% \mathrm{Cl})$ & & & 157 & & & 163 & $100.0 \%$ & $0.05[-0.26,0.35]$ & & \\
\hline \multicolumn{9}{|c|}{$\begin{array}{l}\text { Heterogeneity: } \text { Tau }^{2}=0.03 ; \mathrm{Chi}^{2}=3.15, \mathrm{df}=2(P=0.21) ; \mathrm{I}^{2}=36 \% \\
\text { Test for overall effect: } Z=0.29(P=0.77)\end{array}$} & $\begin{array}{cccc}-1 & -0.5 & 0 & 0.5 \\
\text { Favours control } & \text { Favours in }\end{array}$ & $\begin{array}{c}1 \\
\text { vent }\end{array}$ \\
\hline
\end{tabular}

Fig. 4 Forest plot illustrating the standardised mean difference and 95\% confidence interval of ambulatory ability (6 MWT distance/walking time) at intervention end for rehabilitation interventions with outdoor mobility compared to routine care

intervention $90.5(80.5,98.0)$ comparison: $79.5(40.0,92.5))$ [29]. Rehabilitation interventions which incorporated outdoor mobility were suggestive (confidence interval crosses null) of a small increase in falls-related self-efficacy at 1-3month follow-up compared with routine care (standardised mean difference 0.25 ; 95\% CI: $-0.29,0.78$ ) (Fig. 5). There was substantial heterogeneity in the analysis $\mathrm{I}^{2}=87 \%$. On removal of the RCT by Ziden et al. [24], there was no between-group difference in falls-related self-efficacy at 1-3-month follow-up (standardised mean difference-0.03; $95 \%$ CI: $-0.24,0.18)$ and $\mathrm{I}^{2}=0 \%$. At 12 -month follow-up, there were no between-group differences in falls-related selfefficacy for the study by Resnick et al. [35]. Differences in the Falls Self-Efficacy Scale (Swedish version) observed by Ziden et al. persisted at 6 and 12-month follow-up [25].

\section{Discussion}

\section{Summary of evidence}

We identified 12 papers for 11 RCTs which included outdoor mobility in their rehabilitation intervention for participants after hip fracture. There were methodological concerns related to unblinded participants, personnel, and outcome assessors, and a lack of precision in estimates across included RCTs. Our meta-analyses suggest interventions which include outdoor mobility may be beneficial in terms of outdoor walking and falls-related self-efficacy and not beneficial for walking endurance. However, the RCTs did not provide sufficient detail to replicate the intended outdoor mobility component. Furthermore, most RCTs did not provide detail on the extent to which the outdoor mobility component was actually achieved. Coupled with methodological concerns, we cannot determine the extent to which any potential benefit observed across RCTs may be attributed to an outdoor mobility intervention component.

\section{Interpretation}

The current review identified 7 additional RCTs not included in a previous review of home-based rehabilitation after hip fracture by Wu et al. [13]. We identified the same RCTs by Ziden et al. [24, 25] and Karlsson et al. [28] investigating the effectiveness of interventions on outdoor mobility. Wu et al. proposed no effect on walking outdoors based on these two studies [13]. We adopted a more conservative interpretation of the meta-analysis highlighting the conflicting evidence for effectiveness between the two studies. We also add to the findings of this earlier review by providing results from analysis of both walking endurance and falls-related self-efficacy.

A previous review by Heldmann and colleagues indicated outcome measure selection should be highly specific to the intervention components to reveal benefits attributable to rehabilitation in older patients [11]. For the current review, ambulatory ability and/or falls-related self-efficacy were selected as a primary outcome for 6 of the 11 RCTs identified suggesting outdoor mobility was a peripheral treatment component for half of included RCTs. Indeed, interventions

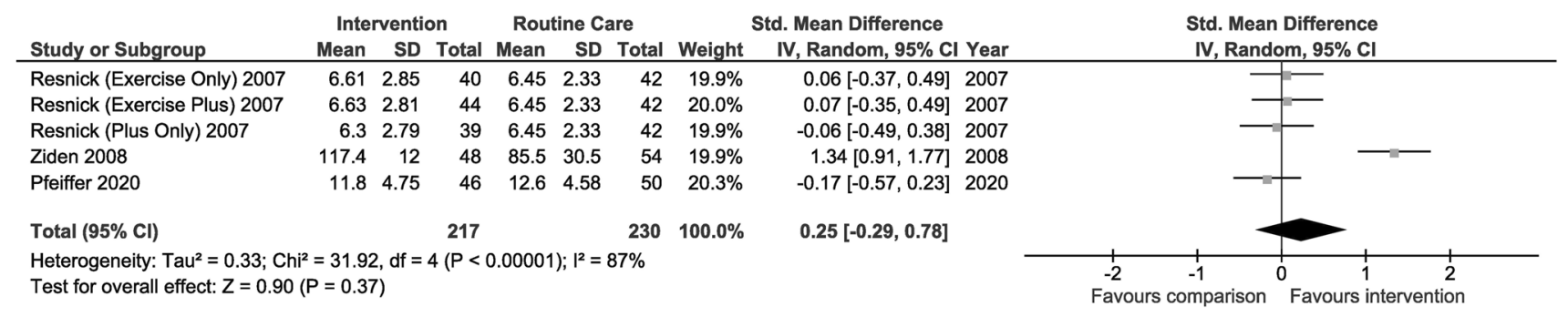

Fig. 5 Forest plot illustrating the standardised mean difference and 95\% confidence interval of falls-related self-efficacy at $1-3$ months followup for rehabilitation interventions with outdoor mobility compared to routine care 
included multiple treatment components many of which do not have a plausible mechanism for changing ambulatory ability or falls-related self-efficacy, e.g. wound care, mediation and nutrition [28]. In addition, most interventions targeted changes in body function/structures through, e.g. resistance training or flexibility (often supervised), as well as changes in activities or participation through (often unsupervised) indoor and outdoor mobility [26, 28, 30, 31, $33,35]$. The peripheral nature of outdoor mobility to these interventions may explain the lack of reported effectiveness on ambulatory ability and falls-related self-efficacy.

Potential benefits in falls-related self-efficacy and/or ambulatory ability were observed for interventions where outdoor mobility was a more central treatment component. The intervention by Ziden et al. focused explicitly on increasing outdoor mobility through physical activity, cognitive behavioural interventions and engagement of family in discharge planning $[24,25]$. The alignment between intervention components and outcomes may explain the positive effect (in terms of earlier recovery of outdoor mobility and increased falls-related self-efficacy) observed compared with routine care [24, 25]. The aerobic training arm of the RCT by Mangione et al. was the only intervention to achieve a clinically meaningful (but not statistically significant) between-group difference for the 6-min walk test at the end of the intervention [27, 37]. The observed difference may be attributed to the relevance of the 6-min walk test to an intervention which focused on $20 \mathrm{~min}$ of indoor and outdoor walking ( $83 \%$ of participants performed outdoor mobility) at 65 to $75 \%$ of age-predicted maximal heart rate [27]. Whilst promising, these interventions were not without methodological concerns. Ziden et al. failed to blind outcome assessors to group allocation which may have led to overestimation of effectiveness [24, 25]. The RCT by Mangione et al. was small with 12 participants in the intervention group and 10 in the control group leading to a lack of precision in outcome estimates. It is therefore not possible to determine whether an intervention with outdoor mobility as a central component leads to benefits in ambulatory ability or fallsrelated self-efficacy after hip fracture.

Half of RCTs included in this review incorporated a psychological treatment component (goal setting and/or motivation) [24, 25, 29, 30, 34, 35]. Evidence from stroke and primary prevention supports a key role of psychological components in interventions targeting outdoor mobility. For patients post-stroke, a large UK multicentre trial, the 'Getting Out of The House Study,' saw a neutral effect of repeated practice of outdoor mobility on outcomes apart from potentially increasing the number of outdoor journeys (secondary study outcome) [38]. The authors noted that the benefit observed was dependent on the treating therapist indicating a role of motivation and feedback [38]. This is in keeping with an implementation intervention in Australia which reported a beneficial effect of targeting the behaviour of community rehabilitation teams to deliver more outdoor journeys for people post-stroke on the proportion of people achieving outdoor mobility after the intervention [39]. An umbrella review of primary prevention interventions pointed to feedback as a core behaviour change treatment component for increasing physical activity among older adults [40]. For the current review, only one study incorporated objective feedback with the use of sensor output for unsupervised indoor mobility to inform coaching during the intervention [32]. This objective feedback was not extended to outdoor mobility and may be targeted in a future intervention study [32].

There is uncertainty over the external validity of many of the studies included in this review to the underlying population of patients with hip fracture. Most excluded potential participants with cognitive impairment (170 of 1868 (9\%) potential participants, where reported) $[24,27,29,32,34$, 35 ], reflecting up to $30 \%$ of the underlying population [41]. Only one RCT included participants' resident in nursing homes [28] where the incidence of hip fracture is high [42]. Moreover, the structure of community-based rehabilitation varies widely regionally, nationally, and internationally. Therefore, it cannot be certain whether results from Australia, Finland, Germany, the Netherlands, Sweden, and the USA may be generalizable to other contexts both within and across countries.

\section{Strengths and limitations}

We used published search terms reviewed by a research librarian. We used broad eligibility criteria with no limitations by characteristics of patients with hip fracture, control group, outcome, length of follow-up or publication date, and used duplicate screening for eligibility and risk of bias, and for a sample set of extracted data to reduce the risk of selection bias. Our broad eligibility criterion for 'outdoor mobility' led to identification of intervention components ranging from goal setting related to outdoor mobility to supervised outdoor walking within a target heart rate range. Whilst providing a summary of the existing evidence on outdoor mobility intervention components, this range may have contributed to the statistical heterogeneity observed in meta-analyses. We did not include protocols, pilot/feasibility studies, nonrandomised studies, conference proceedings and/or RCTs not published in English. We excluded two potentially eligible RCTs that we did not receive responses from the authors to determine whether outdoor mobility was included in their rehabilitation intervention [43, 44]. We excluded RCTs not published in English and secondary analyses of RCTs (including 3 secondary analyses of RCTs included in this review [45, 47]). These exclusions may have 
led to publication bias through the exclusion of evidence relevant to our review question. Finally, we did not assess risk of bias at the outcome level which may have identified additional concerns related to the methodological quality of included studies.

\section{Conclusions}

Previous RCTs incorporated outdoor mobility in their interventions with some indicating a potential benefit in terms of ambulatory ability and/or falls-related self-efficacy after hip fracture. It was not possible to attribute any benefit observed to an outdoor mobility intervention component due to poor reporting of target or achieved outdoor mobility and/or quality of the underlying evidence. Falls-related self-efficacy and the physical ability to mobilise outdoors are critical for patient-reported rehabilitation goals related to participation in social and family networks and activities. Further research on the effectiveness of outdoor mobility interventions after hip fracture on outdoor mobility and known barriers to outdoor mobility (falls-related self-efficacy, falls risk and endurance) is warranted. This research should place outdoor mobility at the centre of an intervention whilst ensuring methodological rigour and addressing challenges for external validity.

Supplementary Information The online version contains supplementary material available at https://doi.org/10.1007/s11657-021-00963-0.

\begin{abstract}
Author contribution KJS, FCM, CS and SEL contributed to the conception and design of the study. In addition, KJS, LF and KL contributed to the acquisition and the analysis of data. All authors contributed to the interpretation of the analysis. KJS drafted the manuscript. All authors critically revised the manuscript. All authors approved the final version for submission.
\end{abstract}

Data availability Not applicable.

Code availability Not applicable.

\section{Declarations}

Ethics approval Not applicable.

Consent to participate Not applicable.

Consent for publication The manuscript has not been submitted to more than one journal for simultaneous consideration. The manuscript has not been published previously (partly or in full). No data have been fabricated or manipulated to support conclusions. No data, text, or theories by others are presented as if they were the authors' own. Consent to submit has been received from all co-authors before submission. Authors whose names appear on the submission have contributed sufficiently to the scientific work and therefore share collective responsibility and accountability for the results.
Conflict of interest KS received funding from the NIHR Research for Patient Benefit, the Chartered Society of Physiotherapy Charitable Trust and UKRI Future Leaders Fellowship for hip fracture health services research. KS is the current Lead of the International Fragility Fracture Network's Hip Fracture Recovery Research Special Interest Group. CS received funding from the National Institutes of Health Research and Dunhill Medical Trust for research not related to the current study. LF, FCM KL, and SL declare no competing interests.

Open Access This article is licensed under a Creative Commons Attribution 4.0 International License, which permits use, sharing, adaptation, distribution and reproduction in any medium or format, as long as you give appropriate credit to the original author(s) and the source, provide a link to the Creative Commons licence, and indicate if changes were made. The images or other third party material in this article are included in the article's Creative Commons licence, unless indicated otherwise in a credit line to the material. If material is not included in the article's Creative Commons licence and your intended use is not permitted by statutory regulation or exceeds the permitted use, you will need to obtain permission directly from the copyright holder. To view a copy of this licence, visit http://creativecommons.org/licenses/by/4.0/.

\section{References}

1. Royal College of Physicians (2019) National Hip Fracture Database (NHFD) Annual Report 2019. Royal College of Phyisicans, London

2. Haentjens P et al (2010) Meta-analysis: excess mortality after hip fracture among older women and men. Ann Intern Med 152(6):380-390

3. Tang VL et al (2017) Rates of recovery to pre-fracture function in older persons with hip fracture: an observational study. J Gen Intern Med 32(2): 153-158

4. Nanjayan SK et al (2014) Predictors of change in 'discharge destination' following treatment for fracture neck of femur. Injury 45(7):1080-1084

5. Leibson CL et al (2002) Mortality, disability, and nursing home use for persons with and without hip fracture: a population-based study. J Am Geriatr Soc 50(10):1644-1650

6. Dreinhofer KE et al (2018) A global call to action to improve the care of people with fragility fractures. Injury 49(8):1393-1397

7. Griffiths F et al (2015) Evaluating recovery following hip fracture: a qualitative interview study of what is important to patients. BMJ Open 5(1):e005406

8. Taylor NF, Barelli C, Harding KE (2010) Community ambulation before and after hip fracture: a qualitative analysis. Disabil Rehabil 32(15):1281-1290

9. Visschedijk J et al (2010) Fear of falling after hip fracture: a systematic review of measurement instruments, prevalence, interventions, and related factors. J Am Geriatr Soc 58(9):1739-1748

10. Goubar A, M.C., Potter C, Kones GD, Sackley C, Ayis S, Sheehan $\mathrm{KJ}, 30$-day survival and recovery after hip fracture by mobilisation timing and dementia: a UK database study. Bone and Joint Journal, 2021. In Press.

11. Heldmann P et al (2019) Early inpatient rehabilitation for acutely hospitalized older patients: a systematic review of outcome measures. BMC Geriatr 19(1):189

12. Crotty, M., et al., Rehabilitation interventions for improving physical and psychosocial functioning after hip fracture in older people. Cochrane Database Syst Rev, 2010(1): p. CD007624. 
13. Wu D, Zhu X, Zhang S (2018) Effect of home-based rehabilitation for hip fracture: a meta-analysis of randomized controlled trials. J Rehabil Med 50(6):481-486

14. Daniela K, Ehrhardt N (2020) Out-of-home mobility and social participation of older people: a photo-based ambulatory assessment study. Journal of Population Ageing 13:547-560

15. McDonough CM et al (2021) Physical therapy management of older adults with hip fracture. J Orthop Sports Phys Ther 51(2):CPG1-CPG81

16. National Clinical Guideline Centre. The management of hip fracture in adults. London: National Clinical Guidelines Centre, 2011. http://www.ncgc.ac.uk. Accessed 01/06/21

17. Liberati A et al (2009) The PRISMA statement for reporting systematic reviews and meta-analyses of studies that evaluate health care interventions: explanation and elaboration. PLoS Med 6(7):e1000100

18. World Health Organisation. Rehabilitation in health systems. 2017: http://apps.who.int/iris/bitstream/handle/10665/254506/ 9789241549974-eng.pdf;jsessionid=18DF8F518ED956AA5FF9 D8772E365298? sequence $=1$. [Accessed on March $5^{\text {th }}$ 2021]

19. Sheehan KJ et al (2019) Inequity in rehabilitation interventions after hip fracture: a systematic review. Age Ageing 48(4):489-497

20. McHugh ML (2012) Interrater reliability: the kappa statistic. Biochem Med (Zagreb) 22(3):276-282

21. Lamb SE et al (2011) Reporting of complex interventions in clinical trials: development of a taxonomy to classify and describe fall-prevention interventions. Trials 12:125

22. Higgins JPT, et al (2011) The Cochrane Collaboration's tool for assessing risk of bias in randomised trials. BMJ. 343.

23. Cohen, J. Statistical power analysis for the behavioral sciences., ed. Erlbaum. 1988, Hillsdale, NJ.

24. Ziden L, Frandin K, Kreuter M (2008) Home rehabilitation after hip fracture. A randomized controlled study on balance confidence, physical function and everyday activities. Clin Rehabil 22(12):1019-33

25. Ziden L, Kreuter M, Frandin K (2010) Long-term effects of home rehabilitation after hip fracture - 1-year follow-up of functioning, balance confidence, and health-related quality of life in elderly people. Disabil Rehabil 32(1):18-32

26. Pfeiffer $\mathrm{K}$ et al (2020) Effects of an intervention to reduce fear of falling and increase physical activity during hip and pelvic fracture rehabilitation. Age Ageing 49(5):771-778

27. Mangione KK et al (2005) Can elderly patients who have had a hip fracture perform moderate- to high-intensity exercise at home? Phys Ther 85(8):727-739

28. Karlsson A et al (2016) Effects of geriatric interdisciplinary home rehabilitation on walking ability and length of hospital stay after hip fracture: a randomized controlled trial. J Am Med Dir Assoc 17(5):464 e9-464 e15

29. Crotty $\mathrm{M}$ et al (2002) Early discharge and home rehabilitation after hip fracture achieves functional improvements: a randomized controlled trial. Clin Rehabil 16(4):406-413

30. Salpakoski A et al (2014) Effects of a multicomponent homebased physical rehabilitation program on mobility recovery after hip fracture: a randomized controlled trial. J Am Med Dir Assoc 15(5):361-368

31. Hauer $\mathrm{K}$ et al (2002) Intensive physical training in geriatric patients after severe falls and hip surgery. Age Ageing 31(1):49-57
32. Pol MC et al (2019) Effectiveness of sensor monitoring in a rehabilitation programme for older patients after hip fracture: a threearm stepped wedge randomised trial. Age Ageing 48(5):650-657

33. Magaziner $\mathbf{J}$ et al (2019) Effect of a multicomponent homebased physical therapy intervention on ambulation after hip fracture in older adults: the CAP randomized clinical trial. JAMA 322(10):946-956

34. Orwig DL et al (2011) Delivery and outcomes of a yearlong home exercise program after hip fracture: a randomized controlled trial. Arch Intern Med 171(4):323-331

35. Resnick B et al (2007) Testing the effectiveness of the exercise plus program in older women post-hip fracture. Ann Behav Med 34(1):67-76

36. Mangione K, C.R., Fortinsky R, .Magaziner J,, Improving community ambulation after hip fracture: -results and lessons learned. American Physical Therapy Association, Combined Sections Meeting, Washington, DC, January, 2019.

37. Bohannon RW, Crouch R (2017) Minimal clinically important difference for change in 6-minute walk test distance of adults with pathology: a systematic review. J Eval Clin Pract 23(2):377-381

38. Logan PA et al (2014) Rehabilitation aimed at improving outdoor mobility for people after stroke: a multicentre randomised controlled study (the Getting out of the House Study). Health Technol Assess 18(29):vii-viii-1 113

39. McCluskey A, Middleton S (2010) Increasing delivery of an outdoor journey intervention to people with stroke: a feasibility study involving five community rehabilitation teams. Implement Sci 5:59

40. Zubala A et al (2017) Promotion of physical activity interventions for community dwelling older adults: A systematic review of reviews. PLoS ONE 12(7):e0180902

41. Mundi S, Chaudhry H, Bhandari M (2014) Systematic review on the inclusion of patients with cognitive impairment in hip fracture trials: a missed opportunity? Can J Surg 57(4):E141-E145

42. Berry SD et al (2016) Incidence of hip fracture in U.S. nursing homes. J Gerontol A Biol Sci Med Sci 71(9):1230-4

43. Crotty M et al (2019) Should we provide outreach rehabilitation to very old people living in nursing care facilities after a hip fracture? A randomised controlled trial Age Ageing 48(3):373-380

44. Suwanpasu SAY, Jitapanya C (2014) Post-surgical physical activity enhancing program for elderly patients after hip fracture: a randomized controlled trial. Asian Biomed 8(4):525-532

45. Karlsson A et al (2020) Effects of geriatric interdisciplinary home rehabilitation on independence in activities of daily living in older people with hip fracture: a randomized controlled trial. Arch Phys Med Rehabil 101(4):571-578

46. Turunen K et al (2017) Physical activity after a hip fracture: effect of a multicomponent home-based rehabilitation program-a secondary analysis of a randomized controlled trial. Arch Phys Med Rehabil 98(5):981-988

47. Edgren $\mathbf{J}$ et al (2015) Effects of a home-based physical rehabilitation program on physical disability after hip fracture: a randomized controlled trial. J Am Med Dir Assoc 16(4):350 e1 7

Publisher's note Springer Nature remains neutral with regard to jurisdictional claims in published maps and institutional affiliations. 